\title{
Stochastic Properties of the Weighted Hazard Rate Order
}

\author{
S. Izadkhah, M. Amini* and G. R. Mohtashami Borzadaran \\ Department of Statistics, Ordered and Spatial Data Center of Excellence \\ Faculty of Mathematical Sciences, Ferdowsi University of Mashhad, Mashhad, Iran \\ Emails:Sa_ei924@stu-mail.um.ac.ir,m-amini@um.ac.ir,grmohtashami@um.ac.ir
}

\author{
Received 14 August 2014 \\ Accepted 3 February 2015
}

\begin{abstract}
The hazard rate order between two weighted distributions with a given weight function is considered as a new stochastic order called weighted hazard rate order. The influences of biased sampling methods on properties of the hazard rate order are usefully pointed out with this weighted order. We first obtain some basic properties of the weighted hazard rate order and then develop some useful preservation properties of it with respect to monotone transformations, mixture of distributions, convolution of distributions, order statistics and residual lifetime of distributions. A few examples are also presented to show the usefulness of the results achieved in the paper.
\end{abstract}

Keywords: Weighted distribution; hazard rate order;preservation; shock models; mixture; stochastic orders.

\section{Introduction}

Weighted distributions are useful to fit to data in situations where the original distribution of the data is naturally unobserved. Patil and Rao [19] identified various situations in which weighted distributions are applicable. Over the past years, weighted distributions have been intuitively used in applied probability and statistics to investigate some statistical properties and inference procedures. For some recent works in applied probability we refer the readers to Unnikrishnan and Sunoj [21], Navarro et al. [16], Alavi and Chinipardaz [1], Li et al. [12] and Izadkhah et al. [7]; and for some recent works in applied statistics we refer the readers to Cutillo et al. [5], Feizjavadian and Hashemi [6] and Karimi and Alavi [9] among others. In particular, reliability analysis of the weighted distributions has received much attention in the literature in the recent past decades (see, for instances, Nanda and Jain [15], Navarro et al. [17], Bartoszewicz and Skolimowska [3], Błazej [4] and Kayid et al. [11]). In reliability and survival analysis, when data are unknowingly sampled from a weighted distribution as opposed to the parent distribution, the survival function, the hazard rate function, and

*Corresponding author 
the mean residual life function may be underestimated or overestimated depending on the form of the weight function.

In the current investigation we study some basic properties of the hazard rate order of weighted distributions including some useful preservation properties that are ordinarily considered in the study of the well-known stochastic orders in the literature. The paper is organized as follows. In section 2, we introduce some notations and preliminaries as they are needed throughout the paper. In section 3, we discuss some characterizations of the weighted hazard rate order, some interrelations of this stochastic order and some connections of this stochastic order with other well-known stochastic orders. A number of preservation properties of the weighted hazard rate order under some reliability operations such as monotonic transformation, mixture, and convolution are obtained in Section 4. In that section, preservation properties of the weighted hazard rate order under order statistics and under residual lifetime of distributions are studied. Throughout the paper, we will use the terms "increasing" and "decreasing" in place of non-decreasing and non-increasing, respectively. We also assume that all ratios, integrals and expectations exist and they are well defined wherever they appear. We will take $u(x)=1$, for all $x \geq 0$, and $u(x)=0$, for all $x<0$. Set $\mathbb{R}=(-\infty,+\infty)$ and $\mathbb{R}^{+}=(0,+\infty)$.

\section{Notations, terminology, and preliminaries}

Let $X$ be a univariate random variable (rv) with distribution function (df) $F$ and probability density function (pdf) $f$. Then, the weighted version of $X$ with a non-negative weight function $w$ for which $0<E[w(X)]<\infty$, is denoted by $X_{w}$ which has the pdf

$$
f_{w}(x)=\frac{w(x) f(x)}{E[w(X)]}
$$

and its df is given by

$$
\begin{aligned}
F_{w}(x) & =\int_{-\infty}^{x} \frac{w(u)}{E[w(X)]} d F(u) \\
& =\frac{E(w(X) \mid X \leq x)}{E[w(X)]} F(x) .
\end{aligned}
$$

Let $Y$ be a random variable with df $G$ and pdf $g$, and also let $\bar{F}=1-F$ and $\bar{G}=1-G$ be the survival functions of $X$ and $Y$, respectively. When $X$ and $Y$ are absolutely continuous random variables the supports of them are given by $S_{X}=\{x \in \mathbb{R}: f(x)>0\}$ and $S_{Y}=\{y \in \mathbb{R}: g(y)>0\}$, respectively. Suppose that $X_{w}$ and $Y_{w}$ are weighted versions of $X$ and $Y$, respectively. Then, their survival functions are, respectively, given by

$$
\bar{F}_{w}(x)=\frac{B_{X}(x) \bar{F}(x)}{E(w(X))}, \quad \bar{G}_{w}(y)=\frac{B_{Y}(y) \bar{G}(y)}{E(w(Y))}
$$

and their distribution functions are, respectively, given by

$$
F_{w}(x)=\frac{A_{X}(x) F(x)}{E(w(X))}, \quad \text { and } \quad G_{w}(y)=\frac{A_{Y}(y) G(y)}{E(w(Y))},
$$


where $A_{X}(x)=E(w(X) \mid X \leq x)$ and $B_{X}(x)=E(w(X) \mid X>x)$, which are given by

$$
A_{X}(x)=\frac{1}{F(x)} \int_{-\infty}^{x} w(u) f(u) d u, \quad \text { and } \quad B_{X}(x)=\frac{1}{\bar{F}(x)} \int_{x}^{\infty} w(u) f(u) d u,
$$

for all $x \in \mathbb{R}$. Similarly, $A_{Y}$ and $B_{Y}$ are defined. The weighted distribution with weight function $w(x)=x$ is called length-biased distribution. Here, we list some important weight functions used in the literature (cf. Patil et al. [18]).

- $w(x)=x^{\beta}, x \in \mathbb{R}^{+}, \beta \in \mathbb{R}^{+}$.

- $w(x)=e^{\beta x}, x \in \mathbb{R}, \beta \in \mathbb{R}$.

- $w(x)=\beta^{x}, x \in \mathbb{R}, \beta \in \mathbb{R}^{+}$.

- $w(x)=x+1, \alpha x+\beta, x \in \mathbb{R}^{+}, \alpha, \beta \in \mathbb{R}^{+}$.

- $w(x)=(\alpha x+\beta) /(\delta x+\gamma)$, for all allowable values $\alpha, \beta, \delta$ and $\gamma$.

- $w(x)=H(x)$, where $H$ is the df of a rv $Z$.

- $w(x)=\bar{H}(x)$, where $\bar{H}$ is the sf of a rv $Z$.

- $w(x)=u(\beta-x), x \in \mathbb{R}, \beta \in \mathbb{R}$ such that $F(\beta)>0$.

- $w(x)=1-u(\beta-x), x \in \mathbb{R}, \beta \in \mathbb{R}$ such that $\bar{F}(\beta)>0$.

Note that when the weight function $w(x)=x^{\beta}$ is chosen, the rv $X_{w}$ is said to have a size-biased distribution of order $\beta$. When $w(x)=u(\beta-x)$, the $\operatorname{rv} X_{w}$ is equal in distribution with $(X \mid X \leq \beta)$ and when $w(x)=1-u(\beta-x)$ the $X_{w}$ is equal in distribution with $(X \mid X>\beta)$. Therefore, the study of truncated distributions can be considered under the framework of the weighted distributions. The following stochastic orders are defined according to Shaked and Shanthikumar [20]. Let $X$ and $Y$ be two univariate non-negative rv's with absolutely continuous df's $F$ and $G$, sf's $\bar{F}=1-F$ and $\bar{G}=1-G$, and pdf's $f$ and $g$, respectively. Also, let $r$ and $s$ be the hazard rate (hr) functions of $X$ and $Y$, respectively. Suppose that $S_{X}=S_{Y}=\mathbb{R}^{+}$.

- $X$ is said to be smaller than $Y$ in the likelihood ratio order (denoted by $X \leq_{l r} Y$ ) if $g(x) / f(x)$ is increasing in $x \in \mathbb{R}^{+}$.

- $X$ is said to be smaller than $Y$ in the usual stochastic order (denoted by $X \leq_{s t} Y$ ) if $\bar{F}(x) \leq$ $\bar{G}(x)$, for all $x \in \mathbb{R}^{+}$, or equivalently if $E(\phi(X)) \leq E(\phi(Y))$, for all increasing functions $\phi$.

- $X$ is said to be smaller than $Y$ in the hazard rate order (denoted by $X \leq_{h r} Y$ ) if $r(x)=$ $f(x) / \bar{F}(x) \geq g(x) / \bar{G}(x)=s(x)$, for all $x \in \mathbb{R}^{+}$, or equivalently if $\bar{G}(x) / \bar{F}(x)$ is increasing in $x \in \mathbb{R}^{+}$.

- $X$ is said to be smaller than $Y$ in the reversed hazard rate order (denoted by $X \leq_{r h} Y$ ) if $f(x) / F(x) \leq g(x) / G(x)$, for all $x \in \mathbb{R}^{+}$, or equivalently if $G(x) / F(x)$ is increasing in $x \in \mathbb{R}^{+}$.

- $X$ is said to be smaller than $Y$ in the mean residual life order (denoted by $X \leq_{m r l} Y$ ) if $E(X-x \mid X>x) \leq E(Y-x \mid Y>x)$, for all $x \in \mathbb{R}^{+}$.

The above stochastic orders are connected to each other as follows:

$$
\begin{gathered}
X \leq_{l r} Y \Longrightarrow X \leq_{h r} Y \Longrightarrow X \leq_{m r l} Y \\
\Downarrow \\
\Downarrow \\
X \leq_{r h} Y \Longrightarrow X \leq_{s t} Y
\end{gathered}
$$


Two aging classes of life distributions are defined below.

- The lifetime rv $X$ with hazard rate $r(x)=f(x) / \bar{F}(x)$ is said to be IFR (DFR) whenever $h(x)$ is increasing (decreasing) in $x \in S_{X}$, or equivalently if $\bar{F}$ is a log-concave (log-convex) function on $S_{X}$.

- The lifetime rv $X$ with reversed hazard rate $\widetilde{r}(x)=f(x) / F(x)$ is said to be DRHR whenever $\widetilde{r}(x)$ is decreasing in $x \in S_{X}$, or equivalently if $F$ is a log-concave function on $S_{X}$.

Next, we give some other notions which are needed among the paper.

Definition 2.1: A function $\phi: A \rightarrow \mathbb{R}$ is dispersive on the interval $A \subseteq \mathbb{R}$ if $\phi(x)-x$ is increasing in $\mathrm{x}$ on $A$.

Definition 2.2: A function $h:[0, \infty) \rightarrow \mathbb{R}$ is said to be star-shaped if for all $0 \leq \lambda \leq 1$ and $x \geq 0$, $h(\lambda x) \leq \lambda h(x)$. It is well-known (e.g., Marshall and Olkin [13], p.453) that $h$ is star-shaped if, and only if, $h(0) \leq 0$ and $h(x) / x$ is increasing for $x \in \mathbb{R}^{+}$.

Definition 2.3: A non-negative measurable function $h(x, y)$ is said to be totally positive of order 2 (abbreviated by $\left.T P_{2}\right)$ in $(x, y) \in A \times B$, if $h\left(x_{1}, y_{1}\right) h\left(x_{1}, y_{2}\right) \geq h\left(x_{2}, y_{1}\right) h\left(x_{1}, y_{2}\right)$, for every $x_{1} \leq x_{2} \in A$ and $y_{1} \leq y_{2} \in B$, where $A$ and $B$ are two subsets of $\mathbb{R}$.

\section{Basic properties}

Firstly, we introduce the weighted hazard rate order and then we study some basic properties of this stochastic order. We also give an example of special weighted distributions. The role of the hazard rate function is well-known in reliability and survival analysis as the hazard rate is very useful in the study of systems. The hazard rate order is also of interest in risk theory and survival analysis. On the other hand, there are situations that lifetime data are not recorded according to the original distribution but according to a weighted distribution. Therefore, it is a natural extension to consider the hazard rate comparison of the weighted distributions instead of the comparison based on the original distributions. This results in establishing a more flexible comparison of lifetime random variables based on the hazard rate function in the sense that observations are not identically distributed as they are coming from the weighted version of the underlying parent distribution. We consider the following definition.

Definition 3.1: The lifetime random variable $X$ is said to be smaller than the lifetime random variable $Y$ in the weighted hazard rate order with weight function $w$ (denoted by $X \leq_{w h r}^{(w)} Y$ ) whenever $X_{w} \leq_{h r} Y_{w}$.

Equivalent conditions for the weighted hazard rate order are stated in the next result. We omit the proof of this result as it is straightforward.

Proposition 3.1: $X \leq_{w h r}^{(w)} Y$ holds if, and only if, one of the following conditions holds:

(i). $\frac{B_{X}(x)}{B_{Y}(x)} \leq \frac{r(x)}{s(x)}$, for all $x \in \mathbb{R}^{+}$. 
(ii). $\int_{x}^{+\infty} w(u) d W(u, x) \geq 0$, for all $x \in \mathbb{R}^{+}$, where $d W(u, x)=[g(u) f(x)-g(x) f(u)] d u$.

We now establish an interrelation property of the weighted hazard rate order by considering different weight functions. Before stating this result, we need the following technical lemma which is presented without proof.

Lemma 3.1. Let $\left(X_{w}\right)_{\phi}$ be the weighted version of $X_{w}$ with the weight function $\phi$ such that $0<$ $E\left(\phi\left(X_{w}\right)\right)<+\infty$. Then, by taking $v(x)=\phi(x) w(x), X_{v}$ and $\left(X_{w}\right)_{\phi}$ are identical distribution, i.e. $X_{v} \stackrel{s t}{=}\left(X_{w}\right)_{\phi}$.

The following theorem strengthens the result of Theorem 9(a) of Bartoszewicz and Skolimowska [3]. The readers are also referred to see Theorem 3.2(b) in Misra et al. [14].

Theorem 3.1. Given two weight functions $w$ and $v, X \leq_{w h r}^{(w)} Y$ implies $X \leq_{w h r}^{(v)} Y$, provided that $v(x) / w(x)$ is increasing in $x$, for all $x$ for which $w(x)>0$.

Proof. Note that $X \leq_{w h r}^{(w)} Y$ means $X_{w} \leq_{h r} Y_{w}$. Because $\phi(x)=v(x) / w(x)$ is increasing in $x$ thus Theorem 9(a) of Bartoszewicz and Skolimowska [3] provides that $\left(X_{w}\right)_{\phi} \leq_{h r}\left(Y_{w}\right)_{\phi}$. By Lemma 3.1 it follows that $\left(X_{w}\right)_{\phi}$ and $\left(Y_{w}\right)_{\phi}$ are identical in distribution with $X_{v}$ and $Y_{v}$, respectively. Hence, we arrive at $X_{v} \leq_{h r} Y_{v}$ which means that $X \leq_{w h r}^{(v)} Y$.

The next result reveals the connection between the whr order and the $h r$ order. It is directly seen from Theorem 3.1 that if we take $w$ as a constant function and $v$ as an increasing function and also if we take $v$ as a constant function and $w$ as a decreasing function, then we derive the following corollary. Note that the result of Corollary 3.1 is known in the literature (see for instances Theorem 2.1 of Nanda and Jain [15], Theorem 9(a) of Bartoszewicz and Skolimowska [3] and Theorem 3.2(b) of Misra et al. [14]).

Corollary 3.1: (i) If $v$ is increasing, then $X \leq_{h r} Y$ implies $X \leq_{w h r}^{(v)} Y$. (ii) If $w$ is decreasing, then $X \leq_{w h r}^{(w)} Y$ implies $X \leq_{h r} Y$.

As a useful result, when dealing with increasing weight functions, the whr order is weaker than the $h r$ order whereas with a decreasing weight function the $w h r$ order is stronger than the $h r$ order. This means that the monotonicity of the weight function is important to establish connections between the $w h r$ order and the $h r$ order.

Remark 3.1: It is noticeable here that since $X \leq_{l r} Y$ yields $X \leq_{h r} Y$ thus in view of Corollary 3.1( $\left.i\right)$, if $w$ is increasing, then $X \leq_{l r} Y$ implies $X \leq_{w h r}^{(w)} Y$. Moreover, because $X \leq_{h r} Y$ gives $X \leq_{m r l} Y$ and also gives $X \leq_{s t} Y$, therefore appealing to Corollary 3.1(ii) if $w$ is decreasing, then $X \leq_{w h r}^{(w)} Y$ implies $X \leq_{m r l} Y$ and furthermore $X \leq_{w h r}^{(w)} Y$ implies $X \leq_{s t} Y$.

We present the following example to accommodate some special weighted distributions.

Example 3.1. Let $X \leq_{w h r}^{(w)} Y$ hold for $w(x)=x^{\alpha}, w(x)=e^{\alpha x}, w(x)=\alpha^{x}, w(x)=u(\alpha-x), w(x)=1-$ $u(\alpha-x)$, each in one time. Then, according to Theorem 3.1 $X \leq_{w h r}^{(v)} Y$ holds with $v(x)=x^{\beta}, v(x)=$ $e^{\beta x}, v(x)=\beta^{x}, v(x)=u(\beta-x), v(x)=1-u(\beta-x)$, respectively, whenever $\alpha \leq \beta$. Let $X \leq_{w h r}^{(w)} Y$ hold with $w(x)=\alpha x+\beta$. Then, using Theorem 3.1 $X \leq_{w h r}^{(v)} Y$ holds with $v(x)=\delta x+\gamma$, when 
$\alpha \gamma \leq \beta \delta$. As another application of Theorem 3.1 suppose that $Z_{1}$ and $Z_{2}$ are two non-negative random variables with df's $H_{1}$ and $H_{2}$ and sf's $\bar{H}_{1}$ and $\bar{H}_{2}$, respectively. Let $X \leq_{w h r}^{(w)} Y$ hold with $w(x)=H_{1}(x)$. Then, $X \leq_{w h r}^{(v)} Y$ holds with $v(x)=H_{2}(x)$, whenever $Z_{1} \leq_{r h} Z_{2}$. Moreover, if $X \leq_{w h r}^{(w)} Y$ holds with $w(x)=\bar{H}_{1}(x)$, then $X \leq_{w h r}^{(v)} Y$ holds with $v(x)=\bar{H}_{2}(x)$, when $Z_{1} \leq_{h r} Z_{2}$.

\section{Preservation properties}

In this section, we mainly investigate the problem of preservation of the weighted hazard rate order under reliability operations of monotonic transformation, mixture and convolution. Afterward, we study some properties of the weighted hazard rate order in the context of order statistics and the residual lifetime. We give some illustrative examples to show the usefulness of the results and in parallel we discuss some useful conclusions which will be resulted from our findings.

\subsection{Monotone transformation}

We discuss here the closure property of the weighted hazard order under monotonic transformation. This result strengthens Theorem 1.B.2 of Shaked and Shanthikumar [16] to the case where the weighted distributions stand in place of the original distributions.

Theorem 4.1. If $\phi$ is a strictly increasing and left continuous function and if $w(\phi(x)) / w(x)$ increases in $x$ on $\{x: w(x)>0\}$, then $X \leq_{w h r}^{(w)} Y$ implies $\phi(X) \leq_{w h r}^{(w)} \phi(Y)$.

Proof. First, let $v$ be a weight function of the form $v(x)=w(\phi(x))$. Then, in view of assumption, by Theorem 3.1, $X_{w} \leq_{h r} Y_{w}$ implies $X_{v} \leq_{h r} Y_{v}$. Because $\phi$ is strictly increasing thus Theorem 1.B.2 in Shaked and Shanthikumar [20] provides that $\phi\left(X_{v}\right) \leq_{h r} \phi\left(Y_{v}\right)$. On the other hand, an application of Theorem 1 in Bartoszewicz [2] yields $\phi\left(X_{v}\right) \stackrel{s t}{=}(\phi(X))_{w}$ and also $\phi\left(Y_{v}\right) \stackrel{s t}{=}(\phi(Y))_{w}$. Thus, it follows that $(\phi(X))_{w} \leq_{h r}(\phi(Y))_{w}$, which means $\phi(X) \leq_{w h r}^{(w)} \phi(Y)$.

We consider the following conclusion of Theorem 4.1 dealing with special weighted distributions.

Corollary 4.1: Suppose that $\phi$ is strictly increasing and left continuous and let one of the following conditions hold:

(i). $w(x)=x^{\beta}, \beta \in \mathbb{R}^{+}$, provided that $\phi$ is a star-shaped function.

(ii). $w(x)=\beta^{x}, \beta>1$, provided that $\phi$ is a dispersive function.

(iii). $w(x)=e^{\beta x}, \beta \in \mathbb{R}^{+}$, provided that $\phi$ is a dispersive function.

(iv). $w(x)=u(\beta-x), \beta \in \mathbb{R}^{+}$, provided that $\phi(x) \leq x$, for all $x \geq 0$.

(v). $w(x)=1-u(\beta-x), \beta \in \mathbb{R}^{+}$, provided that $\phi(x) \leq x$, for all $x \geq 0$.

(vi). $w(x)=H(x)$ where $H$ is df of some non-negative rv $Z$, provided that $\phi(Z) \leq_{r h} Z$.

(vii). $w(x)=\bar{H}(x)$ where $\bar{H}$ is sf of some non-negative $r v Z$, provided that $\phi(Z) \leq_{h r} Z$.

Then, $X \leq_{w h r}^{(w)} Y$ implies $\phi(X) \leq_{w h r}^{(w)} \phi(Y)$.

\subsection{Mixture of distributions}

Consider a family of survival functions $\{\bar{F}(\cdot \mid \theta), \theta \in \chi\}$ where $\chi$ is a subset of the real line $\mathbb{R}$. Let $f(\cdot \mid \theta)$ be the associated pdf of this parametric family and let $X(\theta)$ denote a random variable with 
pdf $f(\cdot \mid \theta)$. For any random variable $\Theta_{i}$ with support in $\chi$, and with distribution function $H_{i}$, and density function $h_{i}$, we denote by $X\left(\Theta_{i}\right)$ the random variable that has survival function $\bar{F}_{i}$ given by

$$
\bar{F}_{i}(x)=\int_{\chi} \bar{F}(x \mid \theta) d H_{i}(\theta), \quad x \in \mathbb{R}, i=1,2 .
$$

In this case, $X\left(\Theta_{i}\right)$ is called a mixture of $X(\theta)$ of the family $\{\bar{F}(\cdot \mid \theta), \theta \in \chi\}$ with respect to $\Theta_{i}$ for each $i=1,2$. Before presenting the first result we state the following lemma which is an extended version of Theorem 1.B.12 in Shaked and Shanthikumar [20]. We omit its proof as it is easily derived.

Lemma 4.1. Let $X_{1}$ and $X_{2}$ be two independent random variables. Then $X_{1} \leq_{w h r}^{(w)} X_{2}$ if, and only if, $E\left(\alpha_{1}\left(X_{1}\right) w\left(X_{1}\right)\right) E\left(\alpha_{2}\left(X_{2}\right) w\left(X_{2}\right)\right) \leq E\left(\alpha_{1}\left(X_{2}\right) w\left(X_{2}\right)\right) E\left(\alpha_{2}\left(X_{1}\right) w\left(X_{1}\right)\right)$ for all functions $\alpha_{1}$ and $\alpha_{2}$ such that $\alpha_{2}$ is non-negative and $\alpha_{1} / \alpha_{2}$ and $\alpha_{2}$ are increasing.

Next, the preservation property of the whr order is established under mixture of a family of distributions. This extends the result of Theorem 1.B.14 of Shaked and Shanthikumar [16] to the context of weighted distributions.

Theorem 4.2. Let $w$ be a weight function such that $E[w(X(\theta))]$ is increasing in $\theta$, let

$$
X\left(\theta_{1}\right) \leq_{w h r}^{(w)} X\left(\theta_{2}\right), \text { for all } \theta_{1} \leq \theta_{2} \in \chi,
$$

and also let

$$
\Theta_{1} \leq_{h r} \Theta_{2}
$$

Then, $X\left(\Theta_{1}\right) \leq_{w h r}^{(w)} X\left(\Theta_{2}\right)$.

Proof. Suppose that $\left(\alpha_{1}, \alpha_{2}\right)$ is an arbitrary pair of functions satisfying conditions of Lemma 4.1. Set $\eta_{i}(\theta)=E\left(\alpha_{i}(X(\theta)) w(X(\theta))\right)$, for $i \in\{1,2\}$ and $\theta \in \chi$. Using Lemma 4.1, (4.2) provides that $\eta_{1}\left(\theta_{1}\right) \eta_{2}\left(\theta_{2}\right) \leq \eta_{1}\left(\theta_{2}\right) \eta_{2}\left(\theta_{1}\right)$, for all $\theta_{1} \leq \theta_{2} \in \chi$, which means that $\eta_{1} / \eta_{2}$ is increasing. Denoting by $X_{w}(\theta)$ the weighted version of $X(\theta)$ with weight function $w$, it is not hard to see that $\eta_{2}(\theta)=$ $E\left(\alpha_{2}\left(X_{w}(\theta)\right)\right) E(w(X(\theta)))$, for each $\theta \in \chi$. Because of (4.2), $X_{w}(\theta)$ is stochastically increasing in $\theta$, i.e., for any increasing function $\alpha_{2}, E\left(\alpha_{2}\left(X_{w}(\theta)\right)\right)$ increases in $\theta$. Therefore, by assumption $\eta_{2}$ is non-negative and increasing. Now, applying Theorem 1.B.14 of Shaked and Shanthikumar [20] with the pair $\left(\eta_{1}, \eta_{2}\right)$ to (4.3) provides that $E\left(\eta_{1}\left(\Theta_{1}\right)\right) E\left(\eta_{2}\left(\Theta_{2}\right)\right) \leq E\left(\eta_{1}\left(\Theta_{2}\right)\right) E\left(\eta_{2}\left(\Theta_{1}\right)\right)$. Noting the fact that $E\left(\eta_{i}\left(\Theta_{j}\right)\right)=E\left(\alpha_{i}\left(X\left(\Theta_{j}\right)\right) w\left(X\left(\Theta_{j}\right)\right)\right), i, j \in\{1,2\}$, in which the expectations are with respect to $\Theta_{j}$ and $X\left(\Theta_{j}\right)$, respectively, a further application of Lemma 4.1 completes the proof.

In the context of Theorem 4.2 it is worth saying that when $w$ is an increasing function and $X\left(\theta_{1}\right) \leq_{s t}$ $X\left(\theta_{2}\right)$, for all $\theta_{1} \leq \theta_{2} \in \chi$, then by definition $E\left[w\left(X\left(\theta_{1}\right)\right)\right] \leq E\left[w\left(X\left(\theta_{2}\right)\right)\right]$, for all $\theta_{1} \leq \theta_{2} \in \chi$, i.e., $E[w(X(\theta))]$ is increasing in $\theta \in \chi$. We now consider the following example.

Example 4.1. Consider the proportional hazard rate family of $X(\theta)$ with survival function $\bar{F}(x \mid \theta)=$ $[\bar{F}(x)]^{\theta}, \theta \in \chi=(0, \infty)$. Obviously, this family is stochastically increasing in $\theta$ with respect to the hr order, i.e., $X\left(\theta_{1}\right) \leq{ }_{h r} X\left(\theta_{2}\right)$, for all $\theta_{1} \leq \theta_{2} \in \chi$. If $w$ is an increasing weight function, then according to Corollary 3.1 it holds that $X\left(\theta_{1}\right) \leq_{w h r}^{(w)} X\left(\theta_{2}\right)$, for all $\theta_{1} \leq \theta_{2} \in \chi$. In addition, because the family of $X(\theta)$ is stochastically increasing thus $E[w(X(\theta))]$ is increasing in $\theta \in \chi$. Hence according to Theorem 4.2, if $\Theta_{1} \leq_{h r} \Theta_{2}$, then $X\left(\Theta_{1}\right) \leq_{w h r}^{(w)} X\left(\Theta_{2}\right)$. 
In Theorem 4.2, by making a stronger stochastic comparison in (4.3) we could get the preservation property of the weighted hazard rate order under mixture without considering any condition on the weight function. The following result clarifies the issue.

Theorem 4.3. Let $w$ be any arbitrary weight function such that

$$
X\left(\theta_{1}\right) \leq_{w h r}^{(w)} X\left(\theta_{2}\right), \text { for all } \theta_{1} \leq \theta_{2} \in \chi,
$$

and also let

$$
\Theta_{1} \leq_{l r} \Theta_{2}
$$

Then, $X\left(\Theta_{1}\right) \leq_{w h r}^{(w)} X\left(\Theta_{2}\right)$.

Proof. In view of the proof of Theorem 4.2, $X\left(\Theta_{1}\right) \leq_{w h r}^{(w)} X\left(\Theta_{2}\right)$ holds if and only if $\int_{\chi} h_{i}(\theta) \phi(x, \theta) d \theta$ is $T P_{2}$ as a function of $i \in\{1,2\}$, and of $\theta \in \chi$, where $h_{i}$ is the pdf of $\Theta_{i}, i=1,2$. As discussed there, because of (4.4), $\phi$ is $T P_{2}$ in $(x, \theta) \in \mathbb{R}^{+} \times \chi$ and because of (4.5), $h_{i}(\theta)$ is $T P_{2}$ in $(i, \theta) \in\{1,2\} \times \chi$. An application of the well-known general composition formula of Karlin [10] completes the proof.

\subsection{Convolution of distributions}

Here we discuss the preservation property of the weighted hazard rate order under the convolution of distributions. This extends Lemma 1.B.3 of Shaked and Shanthikumar [20] to a more general setting arising from the weighted distributions.

Theorem 4.4. Let $w(x)$ be increasing and log-convex for $x \geq 0$ and let $Y$ be a non-negative IFR random variable which is independent of $X_{1}$ and $X_{2}$. If $X_{1} \leq_{w h r}^{(w)} X_{2}$ then $X_{1}+Y \leq_{w h r}^{(w)} X_{2}+Y$.

Proof. Assume that $\left(\alpha_{1}, \alpha_{2}\right)$ is a pair of functions admitting the stated conditions of Lemma 4.1. Denote $\eta_{i}(x)=E\left(\alpha_{i}(x+Y) \frac{w(x+Y)}{w(x)}\right)$, for $i=1,2$. Because $Y$ is IFR, we have $Y+x_{1} \leq_{h r} Y+x_{2}$, for all $x_{1} \leq x_{2} \in \mathbb{R}^{+}$. Now, since $w$ is increasing, by Corollary 3.1(i) it follows that $Y+x_{1} \leq_{w h r}^{(w)}$ $Y+x_{2}$, for all $x_{1} \leq x_{2} \in \mathbb{R}^{+}$. Using Lemma 4.1 it follows that $\eta_{1}\left(x_{1}\right) \eta_{2}\left(x_{2}\right) \leq \eta_{1}\left(x_{2}\right) \eta_{2}\left(x_{1}\right)$, for all $x_{1} \leq x_{2} \in \mathbb{R}^{+}$, which is equivalent to $\eta_{1} / \eta_{2}$ being increasing. By assumption $w(x+y) / w(x)$ is increasing in $x \geq 0$, for all $y \geq 0$, and also we know that $\alpha_{2}$ is non-negative and increasing. This readily concludes that $\eta_{2}$ is non-negative and increasing. As $X_{j}, j=1,2$ is independent of $Y$, we can write for any $i, j \in\{1,2\}$

$$
\begin{aligned}
E\left(\alpha_{i}\left(X_{j}+Y\right) w\left(X_{j}+Y\right)\right) & =E\left(E\left(\alpha_{i}\left(X_{j}+Y\right) w\left(X_{j}+Y\right) \mid X_{j}\right)\right) \\
& =E\left(\eta_{i}\left(X_{j}\right) w\left(X_{j}\right)\right)
\end{aligned}
$$

which by a further application of Lemma 4.1 to $X_{1} \leq_{w h r}^{(w)} X_{2}$ completes the proof.

We now extend Theorem 4.4 to the case of higher order convolutions.

Theorem 4.5. Let $w$ be an increasing log-convex function and let $\left(X_{i}, Y_{i}\right), i=1,2, \ldots, n$, be independent pairs of random variables such that $X_{i} \leq_{w h r}^{(w)} Y_{i}, i=1,2, \ldots, n$. If $X_{i}, Y_{i}, i=1,2, \ldots, n$, are all IFR, 
then

$$
\sum_{i=1}^{n} X_{i} \leq_{w h r}^{(w)} \sum_{i=1}^{n} Y_{i}
$$

Proof. We prove the theorem by induction. Obviously, the result is true for $n=1$. Assume the result is true for $p=n-1$; that is

$$
\sum_{i=1}^{n-1} X_{i} \leq_{w h r}^{(w)} \sum_{i=1}^{n-1} Y_{i}
$$

Because of the closure property of the IFR random variables under convolution, $\sum_{i=1}^{n-1} Y_{i}$ is IFR itself and also because $X_{n}$ is independent of both $\sum_{i=1}^{n-1} X_{i}$ and $\sum_{i=1}^{n-1} Y_{i}$, using Theorem 4.4 we get

$$
\begin{aligned}
\sum_{i=1}^{n} X_{i} & =\left(\sum_{i=1}^{n-1} X_{i}+X_{n}\right) \\
& \leq_{w h r}^{(w)}\left(\sum_{i=1}^{n-1} Y_{i}+X_{n}\right) \\
& \leq_{w h r}^{(w)}\left(\sum_{i=1}^{n-1} Y_{i}+Y_{n}\right)=\sum_{i=1}^{n} Y_{i},
\end{aligned}
$$

where the last inequality follows because $X_{n} \leq_{w h r}^{(w)} Y_{n}$, and because $\sum_{i=1}^{n-1} Y_{i}$ is independent of both $X_{n}$ and $Y_{n}$. Theorem 4.4 was applied once again.

In the following example, we consider some well-known weight functions introduced before that are increasing and log-convex. Hence, they are applicable in Theorems 4.4 and 4.5.

Example 4.2. The weight functions $w(x)=e^{\beta x}, \beta \in \mathbb{R}^{+}, w(x)=\beta^{x}, \beta \in(1, \infty)$, and $w(x)=1-$ $u(\beta-x), \beta \in \mathbb{R}$, are each increasing and log-convex in $x \in \mathbb{R}^{+}$.

\subsection{Order statistics}

In this subsection, we develop the weighted hazard rate order between two probability distributions to the weighted hazard rate order among orders statistics arising from two random samples of the probability distributions. This is a generalization of Theorem 1.B.34 of Shaked and Shanthikumar [20] to the case where the weighted distributions stand in place of the original distributions. Before stating the result we present the following useful lemmas. In what follows, let $X_{1}, X_{2}, . ., X_{n}$ be a random sample of absolutely continuous random variables from $F$ and let $X_{1 w}, X_{2 w}, \ldots, X_{n w}$ be another random sample of absolutely continuous random variables from $F_{w}$. Denote by $X_{i: n}$ and $\left(X_{w}\right)_{i: n}$ the $i$ th order statistics in these two samples, respectively, for each $i=1,2, \ldots, n$. The following lemma states that the order statistics from a random sample of the weighted distribution with weight $w$ is equal in distribution to the weighted version of the order statistics from a random sample of the original distribution with a certain form of the weight function.

Lemma 4.2. Let $v$ be a weight function of the form $v(x)=w(x)\left[A_{X}(x)\right]^{i-1}\left[B_{X}(x)\right]^{n-i}$. Then, $\left(X_{w}\right)_{i: n}$ and $\left(X_{i: n}\right)_{v}$ are equal in distribution, i.e. $\left(X_{w}\right)_{i: n} \stackrel{s t}{=}\left(X_{i: n}\right)_{v}$. 
Proof. For each $i=1,2, \ldots, n$ and for all $x \in \mathbb{R}$, the density function of $\left(X_{i: n}\right)_{v}$ is derived by

$$
\begin{aligned}
f_{\left(X_{i: n}\right)_{v}}(x) & =\frac{v(x) f_{X_{i: n}}(x)}{E\left[v\left(X_{i: n}\right)\right]} \\
& =\frac{n !}{(n-i) !(i-1) ! E\left[v\left(X_{i: n}\right)\right]} v(x) f(x) F^{i-1}(x) \bar{F}^{n-i}(x),
\end{aligned}
$$

and the density function of $\left(X_{w}\right)_{i: n}$ is

$$
\begin{aligned}
f_{\left(X_{w}\right)_{i: n}}(x) & =\frac{n !}{(n-i) !(i-1) !} f_{w}(x) F_{w}^{i-1}(x) \bar{F}_{w}{ }^{n-i}(x) \\
& =\frac{n !}{(n-i) !(i-1) ! E^{n}[w(X)]} w(x) f(x)\left[A_{X}(x) F(x)\right]^{i-1}\left[B_{X}(x) \bar{F}(x)\right]^{n-i} .
\end{aligned}
$$

We know that $\left(X_{w}\right)_{i: n}$ and $\left(X_{i: n}\right)_{v}$ are equal in distribution if and only if

$$
v(x) f(x) F^{i-1}(x) \bar{F}^{n-i}(x)=w(x) f(x)\left[A_{X}(x) F(x)\right]^{i-1}\left[B_{X}(x) \bar{F}(x)\right]^{n-i}
$$

which gives the required form of the weight function $v$ as stated.

Lemma 4.3. (Bartoszewicz and Skolimowska [3]) If $v$ is increasing, then $X \leq_{h r} X_{v}$.

Theorem 4.6. Let $X_{i} \leq_{w h r}^{(w)} Y_{i}, i=1,2, \ldots, n$ and let for a fixed $k \in\{1,2, \ldots, n\}$,

$$
\left[\frac{A_{X}(x)}{A_{Y}(x)}\right]^{k-1}\left[\frac{B_{X}(x)}{B_{Y}(x)}\right]^{n-k}
$$

be increasing in $x \in[0, \infty)$, and $\left[A_{X}(x)\right]^{k-1}\left[B_{X}(x)\right]^{n-k}$ be decreasing in $x \in[0, \infty)$. Then, $X_{k: n} \leq_{w h r}^{(w)}$ $Y_{k: n}$.

Proof. Because $X_{k w}$ 's are identically distributed we have by assumption that $X_{k w} \leq_{h r} Y_{k w}, k=$ $1,2, \ldots, n$. By using Theorem 1.B.34 of Shaked and Shanthikumar [16] we get $\left(X_{w}\right)_{k: n} \leq_{h r}\left(X_{w}\right)_{k: n}$, for each fixed $k$. As a result of Lemma 4.2 we can write $\left(X_{w}\right)_{k: n} \stackrel{s t}{=}\left(X_{k: n}\right)_{v_{1}}$ and $\left(Y_{w}\right)_{k: n} \stackrel{s t}{=}\left(Y_{k: n}\right)_{v_{2}}$, in which

$$
v_{1}(x)=w(x)\left[A_{X}(x)\right]^{k-1}\left[B_{X}(x)\right]^{n-k}, \text { and } \quad v_{2}(x)=w(x)\left[A_{Y}(x)\right]^{k-1}\left[B_{Y}(x)\right]^{n-k} .
$$

Thus, it follows that $\left(X_{k: n}\right)_{v_{1}} \leq_{h r}\left(Y_{k: n}\right)_{v_{2}}$. Now, by taking $v(x)=v_{1}(x) / v_{2}(x)$, which by assumption is increasing in $x$, and then using Lemma 3.1 and Corollary 3.1(i) we arrive at $\left(X_{k: n}\right)_{v_{1}} \leq_{h r}\left(Y_{k: n}\right)_{v_{1}}$. By assumption $w(x) / v_{1}(x)$ is increasing in $x$. On using Theorem 2.1 it then follows that $\left(X_{k: n}\right)_{w} \leq{ }_{h r}$ $\left(Y_{k: n}\right)_{w}$ which means $X_{k: n} \leq_{w h r}^{(w)} Y_{k: n}$.

To indicate the usefulness of Theorem 4.6 in recognizing weighted hazard rate order of the order statistics we present the following example which involves truncated distributions.

Example 4.3. Assume that $X_{1}, \ldots, X_{n}$ and $Y_{1}, \ldots, Y_{n}$ are two random samples from distribution functions $F$ and $G$, respectively, such that $X_{i} \leq_{r h} Y_{i}, i=1, \ldots, n$. Assume that $w(x)=u(\beta-x)$, in which 
$\beta$ is such that $F(\beta)>0$ and $G(\beta)>0$. For all $x \geq 0$, we can get

$$
A_{X}(x)=\left\{\begin{array}{ll}
1 & x \leq \beta, \\
\frac{F(\beta)}{F(x)} & x>\beta .
\end{array} A_{Y}(x)= \begin{cases}1 & x \leq \beta, \\
\frac{G(\beta)}{G(x)} & x>\beta .\end{cases}\right.
$$

Therefore, for all $x \geq 0$ and for $k=n$, we have

$$
\left[\frac{A_{X}(x)}{A_{Y}(x)}\right]^{k-1}\left[\frac{B_{X}(x)}{B_{Y}(x)}\right]^{n-k}= \begin{cases}1 & x \leq \beta, \\ \left(\frac{F(\beta)}{G(\beta)} \frac{G(x)}{F(x)}\right)^{n-1} & x>\beta .\end{cases}
$$

which is increasing in $x \geq 0$, since $G(x) / F(x)$ is increasing from $X_{i} \leq_{r h} Y_{i}, i=1, \ldots, n$. On the other hand, for $k=n$,

$$
\left[A_{X}(x)\right]^{k-1}\left[B_{X}(x)\right]^{n-k}= \begin{cases}1 & x \leq \beta \\ \left(\frac{F(\beta)}{F(x)}\right)^{n-1} & x>\beta\end{cases}
$$

is decreasing in $x \geq 0$. Thus, by Theorem 4.6, if $\left(X_{i} \mid X_{i} \leq \beta\right) \leq_{h r}\left(Y_{i} \mid Y_{i} \leq \beta\right), i=1, \ldots, n$; then $\left(X_{n: n} \mid X_{n: n} \leq \beta\right) \leq_{h r}\left(Y_{n: n} \mid Y_{n: n} \leq \beta\right)$.

\subsection{Residual lifetime}

One of the important aspects of lifetime distributions is the family of the residual life distribution which has found many applications over the years. The problem of modeling the residual lifetime of systems and components as well as the original lifetime of those systems and components is very important in reliability theory and survival analysis. This is because in many situations in practice we need to evaluate the future lifetime of used systems that are still alive. Formally, let $X$ be a lifetime random variable with survival function $\bar{F}$. Then, the conditional random variable $X_{t}=(X-t \mid X>t)$, for all $t$ that $\bar{F}(t)>0$, is well-known as the residual lifetime of $X$ at the age $t$ provided that it has survived up to $t$. It can be easily seen that the hr function of $X_{t}$ is shifted above the hr function of $X$, i.e. $r_{t}(x)=r(t+x)$ for all $x \geq 0$ and $t \geq 0$, where $r_{t}$ and $r$ are the hr functions of $X$ and $X_{t}$, respectively. As an evident consequence, we have

$$
X \leq_{h r} Y \Leftrightarrow X_{t} \leq_{h r} Y_{t}, \text { for all } t \geq 0 .
$$

Suppose that $\left(X_{t}\right)_{w}$ is the weighted version of $X_{t}$ with weight function $w$, then since the hr function of $\left(X_{t}\right)_{w}$ is not shifted above the $\mathrm{hr}$ function of $X_{w}$, thus the property of (4.6) does not remain true in general with the weighted hazard order instead of the hazard rate order. However, as the following result demonstrates, this property is satisfied under a suitable condition.

Theorem 4.7. Let $w$ be a log-concave weight function. Then,

$$
X \leq_{w h r}^{(w)} Y \Leftrightarrow X_{t} \leq_{w h r}^{(w)} Y_{t}, \text { for all } t \geq 0 .
$$

Proof. By taking $t=0$ in $X_{t} \leq_{w h r}^{(w)} Y_{t}$ we arrive at $X \leq_{w h r}^{(w)} Y$. To prove the inverse implication we first introduce some notations. Let $\left(X_{w}\right)_{t}=\left(X_{w}-t \mid X_{w}>t\right)$, for all $t$ that $\bar{F}_{w}(t)>0$ and $\left(Y_{w}\right)_{t}=\left(Y_{w}-t \mid\right.$ $Y_{w}>t$ ), for all $t$ that $\bar{G}_{w}(t)>0$, which are the residual lifetimes of $X_{w}$ and $Y_{w}$, respectively. For the weight function $w_{1}(x)=w(t+x)$, let $\left(X_{t}\right)_{w_{1}}$ and $\left(Y_{t}\right)_{w_{1}}$ be the weighted versions of $X_{t}$ and $Y_{t}$, respectively. By definition, $X \leq_{w h r}^{(w)} Y$ implies $X_{w} \leq_{h r} Y_{w}$ and by (4.6) this gives $\left(X_{w}\right)_{t} \leq_{h r}\left(Y_{w}\right)_{t}$, for 
all $t$ for which $\bar{F}_{w}(t)>0$ and $\bar{G}_{w}(t)>0$. Because of Lemma 2.2 in Izadkhah et al. [8], $\left(X_{w}\right)_{t} \stackrel{s t}{=}\left(X_{t}\right)_{w_{1}}$ and $\left(Y_{w}\right)_{t} \stackrel{s t}{=}\left(Y_{t}\right)_{w_{1}}$, for all allowable $t$ 's. Hence, it follows that $\left(X_{t}\right)_{w_{1}} \leq_{h r}\left(Y_{t}\right)_{w_{1}}$, for all $t$. Now, since $w$ is log-concave, $w(x) / w_{1}(x)=w(x) / w(t+x)$ is increasing in $x$, for all $t$. Therefore, using Theorem 3.1, $\left(X_{t}\right)_{w} \leq_{h r}\left(Y_{t}\right)_{w}$, for all $t$, which means $X_{t} \leq_{w h r}^{(w)} Y_{t}$, for all $t$.

In the following example we consider some log-concave weight functions to be applied in Theorem 4.7 .

Example 4.4. The weight functions $w(x)=x^{\beta} ; \beta \in \mathbb{R}^{+}, w(x)=e^{\beta x} ; \beta \in \mathbb{R}, w(x)=\alpha x+\beta ; \alpha, \beta \in$ $\mathbb{R}^{+}, w(x)=(\alpha x+\beta) /(\delta x+\gamma) ; \beta \delta \leq \alpha \gamma, w(x)=H(x)$, when $H$ is $D R H R, w(x)=\bar{H}(x)$, when $H$ is IFR, and $w(x)=u(\beta-x) ; \beta \in \mathbb{R}^{+}$, are each log-concave in $x$.

\section{Acknowledgments}

The authors are grateful to the referee for making some useful discussions and giving some constructive suggestions which have been very useful in improving the paper.

\section{References}

[1] S.M.R. Alavi and R. Chinipardaz, Form-invariance under weighted sampling. Statistics 43 (1) (2009) 81-90.

[2] J. Bartoszewicz, On a representation of weighted distributions. Statistics and Probability Letters 79 (2009) 1690-1694.

[3] J. Bartoszewicz and M. Skolimowska, Preservation of classes of life distributions and stochastic orders under weighting. Statistics and Probability Letters 56 (2006) 587-596.

[4] P. Błazej, Preservation of classes of life distributions under weighting with a general weight function. Statistics and Probability Letters 78 (2008) 3056-3061.

[5] L. Cutillo, I.D. Feis, C. Nikolaidou and T. Sapatinas, Wavelet density estimation for weighted data. Journal of Statistical Planning and Inference 146 (2014) 1-19.

[6] S.H. Feizjavadian and R. Hashemi, Mean residual weighted versus the length-biased Rayleigh distribution. Journal of Statistical Computation and Simulation (2014), DOI: 10.1080/00949655.2014.942306.

[7] S. Izadkhah, A.H. Rezaei Roknabadi and G.R. Mohtashami Borzadaran, Aspects of the mean residual life order for weighted distributions. Statistics 48 (4) (2014) 851-861.

[8] S. Izadkhah, A.H. Rezaei Roknabadi, M. Amini and G.R. Mohtashami Borzadaran, A general approach for preservation of some aging classes under weighting. Communications in Statistics - Theory and Methods 42 (10) (2013) 1899-1909.

[9] M. Karimi and S.M.R. Alavi, The effect of weight function on hypothesis testing in weighted sampling. Journal of Applied Statistics (2014), DOI: 10.1080/02664763.2014.920777.

[10] S. Karlin (1968). Total Positivity. Stanford University Press, Stanford.

[11] M. Kayid, I.A. Ahmad, S. Izadkhah and A.M. Abouammoh, Further results involving the mean time to failure order, and the decreasing mean time to failure class. IEEE Transactions on Reliability 62 (3) (2013) 670-678.

[12] L. Li, L. Yu and T. Hu, Probability inequalities for weighted distributions. Journal of Statistical Planning and Inference 142 (2012) 1272-1278.

[13] A.W. Marshall and I. Olkin (1979). Inequalities: Theory of Majorization and its Applications. New York: Academic Press.

[14] N. Misra, N. Gupta and I.D. Dhariyal, Preservation of some aging properties and stochastic orders by weighted distributions. Communications in Statistics - Theory and Methods 37 (2008) 627-644.

[15] A.K. Nanda and K. Jain, Some weighted distribution results on univariate and bivariate cases. Journal of Statistical Planning and Inference 77 (1999) 169-180. 
[16] J. Navarro, J.M. Ruiz and Y. Del Aguila, Multivariate weighted distributions: a review and some extensions. Statistics 40 (1) (2006) 51-64.

[17] J. Navarro, Y. Del Aguila and J.M. Ruiz, Characterizations through reliability measures from weighted distributions. Statistical Papers 42 (2001) 395-402.

[18] G.P. Patil, C.R. Rao and M.V. Ratnaparkhi, On discrete weighted distributions and their use in model choice for observed data. Communications in Statistics - Theory and Methods 15 (3) (1986) 907-918.

[19] G.P. Patil and C.R. Rao, Weighted distributions and size-biased sampling with applications to wild-life populations and human families. Biometrics 34 (1978) 179-189.

[20] M. Shaked and J.G. Shanthikumar (2007). Stochastic Orders. Springer Verlag, New York.

[21] N. Unnikrishnan and S.M. Sunoj, Form-invariant bivariate weighted models. Statistics 37 (3) (2003) 259-269. 